\title{
SALLY'S QUESTION AND A CONJECTURE OF SHIMODA
}

\author{
SHIRO GOTO, LIAM O'CARROLL, \\ AND FRANCESC PLANAS-VILANOVA
}

\begin{abstract}
In 2007, Shimoda, in connection with a long-standing question of Sally, asked whether a Noetherian local ring, such that all its prime ideals different from the maximal ideal are complete intersections, has Krull dimension at most 2. In this paper, having reduced the conjecture to the case of dimension 3 , if the ring is regular and local of dimension 3 , we explicitly describe a family of prime ideals of height 2 minimally generated by three elements. Weakening the hypothesis of regularity, we find that, to achieve the same end, we need to add extra hypotheses, such as completeness, infiniteness of the residue field, and the multiplicity of the ring being at most 3 . In the second part of the paper, we turn our attention to the category of standard graded algebras. A geometrical approach via a double use of a Bertini theorem, together with a result of Simis, Ulrich, and Vasconcelos, allows us to obtain a definitive answer in this setting. Finally, by adapting work of Miller on prime Bourbaki ideals in local rings, we detail some more technical results concerning the existence in standard graded algebras of homogeneous prime ideals with an (as it were) excessive number of generators.
\end{abstract}

\section{$\S 1$. Introduction}

It is by now a classic result that in a Noetherian local ring, the existence of a uniform bound on the minimal number of generators of all its ideals is equivalent to its Krull dimension being at most 1. In 1978, Sally (see [Sa, p. 52]) extended this result in the following way. Let $(R, \mathfrak{m}, k)$ be a Noetherian local ring. Then there exists an integer $N \geq 1$ such that the minimal number of generators $\mu(I)$ of an ideal $I$ is bounded above by $N$, for any ideal $I$ of $R$, such that $\mathfrak{m}$ is not an associated prime of $I$ if and only if $\operatorname{dim}(R)$, the Krull dimension of $R$, is at most 2. In particular, if $\operatorname{dim}(R) \leq 2$, then there exists a bound on the minimal number of generators of all its

Received October 7, 2011. Revised December 20, 2011. Accepted August 8, 2012.

First published online May 21, 2013.

2010 Mathematics Subject Classification. Primary 13A17; Secondary 13F15.

Planas-Vilanova's work was partially supported by grant MTM 2010-20279-C02-01. 
prime ideals. Sally then remarked that it is an open question whether the converse is true (see [Sa, p. 53]). In other words, if $(R, \mathfrak{m}, k)$ is a Noetherian local ring such that there exists an integer $N \geq 1$ such that $\mu(\mathfrak{p}) \leq N$, for all prime ideals $\mathfrak{p}$ of $R$, is then $\operatorname{dim}(R) \leq 2$ ?

This question has remained open, and not much progress has been made since that time. In 2007, Shimoda [Sh] asked whether a Noetherian local ring, such that all its prime ideals different from the maximal ideal are complete intersections, has Krull dimension at most 2. Observe that, if all the primes other than the maximal ideal are complete intersections, then, in particular, the cardinalities of their sets of minimal generators are bounded above by the Krull dimension of the ring.

Though the question of Shimoda seems easier than that of Sally because its hypothesis is at first sight much stronger, it has proved to be difficult to answer.

For the sake of simplicity, we will call a Noetherian local ring $(R, \mathfrak{m}, k)$ a Shimoda ring if every prime ideal in the punctured spectrum is of the principal class; that is, the minimal number of generators $\mu(\mathfrak{p})$ of every prime ideal $\mathfrak{p}$ of $R, \mathfrak{p} \neq \mathfrak{m}$, is equal to height $(\mathfrak{p})$.

We first observe that one can reduce the conjecture to the consideration of a local unique factorization domain (UFD) $R$ of Krull dimension at most 3 . A positive answer to Shimoda's conjecture then amounts to showing that either $\operatorname{dim}(R)=2$ or else $\operatorname{dim}(R)=3$ and then exhibiting a prime ideal of height 2 minimally generated by three (or more) elements. This question is attractive because of the combination of its simplicity and its seeming difficulty. Indeed, we are not able, up to now, to produce in full generality a noncomplete intersection prime ideal of height 2 in a local UFD of Krull dimension 3 .

However, in Section 2, we are able to give some partial positive answers. First, if the ring $R$ is regular and local of Krull dimension 3, then we explicitly describe a family of prime ideals of height 2 minimally generated by three elements. These ideals are determinantal ideals of $(2 \times 3)$-matrices, and in the geometric case, they are precisely the defining ideals of irreducible affine space monomial curves.

Next, when trying to weaken the hypothesis of regularity, we find that we need to add extra hypotheses, such as completeness, infiniteness of the residue field, and the multiplicity of the ring being at most 3 . In this case, if $\operatorname{dim}(R)=3$, we exhibit an ideal of height 2 minimally generated by three elements which has a minimal prime over it which is not Gorenstein. This 
will enable us to conclude that a Shimoda ring, with these extra hypotheses, has Krull dimension at most 2.

In the second part of the paper we turn our attention to the category of standard graded algebras (with a graded definition of the notion of a Shimoda ring). In Section 3 we find that a geometrical approach via a double use of a Bertini theorem, together with a result of Simis, Ulrich, and Vasconcelos (see [SUV, Theorem 4.6]), allows us to obtain a definitive result. In Section 4, we sketch an adaptation of Miller's arguments in [Mi] to the case of standard graded rings of interest that allows us, under somewhat more technical hypotheses, to produce homogeneous prime ideals requiring an arbitrarily large number of generators, and also allows us to present a mild generalization of the core result of Section 3 .

\section{$\S 2$. The Shimoda conjecture for rings of small multiplicity}

Let $(R, \mathfrak{m}, k)$ be a Noetherian local ring, of Krull dimension $d \geq 1$, and $k=A / \mathfrak{m}$, the residue class field of $R$. For the remainder of this section, we fix the following notation: the ring $R$ will be called a Shimoda ring if every prime ideal in the punctured spectrum is of the principal class; that is, the minimal number of generators $\mu(\mathfrak{p})$ of every prime ideal $\mathfrak{p}$ of $R, \mathfrak{p} \neq \mathfrak{m}$, is equal to height $(\mathfrak{p})$.

REMARK 2.1. Let $(R, \mathfrak{m}, k)$ be a Noetherian local ring of Krull dimension $d \geq 1$. If $d=1, R$ is Shimoda if and only if $R$ is a domain. In particular, a Shimoda ring need not be Gorenstein since $R=k\left[\left[t^{3}, t^{4}, t^{5}\right]\right]$ is a 1-dimensional local domain that is not Gorenstein (see [E, Exercise 21.11] or [Ma2, Exercise 18.8, p. 152]). Moreover, the completion of a Shimoda ring is not necessarily a Shimoda ring, because $A=\left(k[x, y] /\left(y^{2}-x^{2}-x^{3}\right)\right)_{(x, y)}$ is a 1-dimensional Noetherian local domain whose completion is not a domain [E, p. 185].

REMARK 2.2. Let $(R, \mathfrak{m}, k)$ be a Noetherian local ring of Krull dimension $d \geq 2$. If $R$ is Shimoda, then $R$ is a UFD, and the converse holds if $d=2$. Suppose now that $d \geq 2$ and that $R$ is Shimoda. Then $R$ is also CohenMacaulay. Indeed, take $\mathfrak{p}$ a prime ideal with height $(\mathfrak{p})=d-1$. Since $\mu(\mathfrak{p})=$ $\operatorname{height}(\mathfrak{p})$, then $\mathfrak{p}$ is generated by a regular sequence, say, $x_{1}, \ldots, x_{d-1}$ (see $\left[\mathrm{D}\right.$, Remark, p. 203]). Set $y \in \mathfrak{m}, y \notin \mathfrak{p}$. Since $\mathfrak{p}$ is prime, $x_{1}, \ldots, x_{d-1}, y$ is a regular sequence of length $d$.

The purpose of this section is to prove the following result, where $e(R)$ stands for the multiplicity of $R$ with respect to $\mathfrak{m}$. 
THEOREM 2.3. Let $(R, \mathfrak{m}, k)$ be a Shimoda ring of Krull dimension $d \geq 2$. Suppose that, in addition, either

(a) $R$ is regular, or

(b) $R$ is complete, $R \supset k, k$ is infinite, and $e(R) \leq 3$.

Then $d=2$.

Note in passing that when we weaken the hypothesis from regular $(e(R)=1)$ to $e(R) \leq 3$, we need to add the extra hypotheses that $R$ is complete and contains its infinite residue field. Note that in either case, $R$ is a local CohenMacaulay domain, and we use properties of such domains without further mention. We first show how to reduce the dimension of $R$.

REMARK 2.4. Let $(R, \mathfrak{m}, k)$ be a Shimoda ring of Krull dimension $d \geq 2$. Let $a \in \mathfrak{m} \backslash \mathfrak{m}^{2}$. Then $R / a R$ is a Shimoda ring of Krull dimension $d-1$. Moreover,

(a) if $R$ is regular, then $R / a R$ is regular;

(b) if $R$ is complete, then $R / a R$ is complete; if $k$ is infinite, then $a$ can be chosen to be a superficial element and $e(R / a R)=e(R)$.

In particular, in Theorem 2.3, one can suppose that $2 \leq d \leq 3$.

Proof of Remark 2.4. Take $a \in \mathfrak{m} \backslash \mathfrak{m}^{2}$. Given $\mathfrak{p}$, a prime ideal, $\mathfrak{p} \neq \mathfrak{m}$, such that $a \in \mathfrak{p}$, then $a \in \mathfrak{p} \backslash \mathfrak{m p}$. Therefore, $\mu(\mathfrak{p} / a R)=\mu(\mathfrak{p})-1=\operatorname{height}(\mathfrak{p})-1=$ height $(\mathfrak{p} / a R)$, and $R / a R$ is Shimoda of Krull dimension $d-1$. Clearly, if $R$ is regular, $R / a R$ is regular, and if $R$ is complete, $R / a R$ is complete. Moreover, if $k$ is infinite, there exists a superficial element $a \in \mathfrak{m} \backslash \mathfrak{m}^{2}$ and $e(R / a R)=$ $e(R)$ (see, e.g., [HS, Propositions 8.5.7 and 11.1.9]). Finally, suppose that Theorem 2.3 is true for $2 \leq d \leq 3$, and suppose there exists a Shimoda ring $R$ of Krull dimension $d \geq 4$. By successively factoring out appropriate elements, one would get a Shimoda ring of Krull dimension $\bar{d}=3$. But, by Theorem 2.3, in the case $2 \leq d \leq 3$, one would deduce $\bar{d}=2$, a contradiction. Therefore, Shimoda rings of Krull dimension $d \geq 4$ cannot exist, and one has only to prove Theorem 2.3 for the case $2 \leq d \leq 3$.

Let us fix now some notation that will hold for the rest of the section (see $[\mathrm{OP}])$.

Remark 2.5. Let $(R, \mathfrak{m}, k)$ be a Cohen-Macaulay local ring, and let $x_{1}, x_{2}, x_{3}$ be a regular sequence in $R$. Take $a=\left(a_{1}, a_{2}, a_{3}\right) \in \mathbb{N}^{3}$ and $b=$ 
$\left(b_{1}, b_{2}, b_{3}\right) \in \mathbb{N}^{3}$. Let $c=a+b, c=\left(c_{1}, c_{2}, c_{3}\right)$. Let $\mathcal{M}$ be the matrix

$$
\mathcal{M}=\left(\begin{array}{ccc}
x_{1}^{a_{1}} & x_{2}^{a_{2}} & x_{3}^{a_{3}} \\
x_{2}^{b_{2}} & x_{3}^{b_{3}} & x_{1}^{b_{1}}
\end{array}\right),
$$

and let $v_{1}=x_{1}^{c_{1}}-x_{2}^{b_{2}} x_{3}^{a_{3}}, v_{2}=x_{2}^{c_{2}}-x_{1}^{a_{1}} x_{3}^{b_{3}}$, and $D=x_{3}^{c_{3}}-x_{1}^{b_{1}} x_{2}^{a_{2}}$ be the $2 \times 2$ minors of $\mathcal{M}$ up to a change of sign. Consider $I=I_{2}(\mathcal{M})=\left(v_{1}, v_{2}, D\right)$, the determinantal ideal generated by the $2 \times 2$ minors of $\mathcal{M}$. Then $I$ is a non-Gorenstein height-unmixed ideal of height 2 , minimally generated by three elements. For simplicity, $I$ will be called the $H N$ ideal (from HerzogNorthcott) associated to $x_{1}, x_{2}, x_{3}$ and $a, b \in \mathbb{N}^{3}$. We will also set $m_{1}=c_{2} c_{3}-$ $a_{2} b_{3}, m_{2}=c_{1} c_{3}-a_{3} b_{1}, m_{3}=c_{1} c_{2}-a_{1} b_{2}$, and $m(a, b)=\left(m_{1}, m_{2}, m_{3}\right) \in \mathbb{N}^{3}$. Note that $m_{i} \geq 3$.

Proof of Remark 2.5. That $I$ is height-unmixed of height 2 follows from [OP, Propositions 4.2 and 2.2]. That $I$ is minimally generated by three elements and is non-Gorenstein follows from [OP, Lemma 6.1] and its proof, where a nonsymmetric minimal resolution of $R / I$ is shown.

The proof of Theorem 2.3 is divided into two parts. We first state the regular case.

Proposition 2.6. Let $(R, \mathfrak{m}, k)$ be a regular local ring of Krull dimension 3. Let $x_{1}, x_{2}, x_{3}$ be a regular system of parameters in $R$, and let $a, b \in$ $\mathbb{N}^{3}$. Let I be the HN ideal associated to $x_{1}, x_{2}, x_{3}$ and $a, b$. If $\operatorname{gcd}(m(a, b))=1$, then $I$ is prime. In particular, $R$ is not Shimoda.

Taking $a=(1,1,1)$ and $b=(2,1,1)$ in Proposition 2.6, one obtains the following result.

Corollary 2.7. Let $(R, \mathfrak{m}, k)$ be a regular local ring of Krull dimension 3 . Let $x_{1}, x_{2}, x_{3}$ be a regular system of parameters in $R$. Then $I=$ $\left(x_{1}^{3}-x_{2} x_{3}, x_{2}^{2}-x_{1} x_{3}, x_{3}^{2}-x_{1}^{2} x_{2}\right)$ is a height 2 prime ideal minimally generated by three elements.

For the case of small multiplicity, we have the following. As above, we consider the HN ideal associated to $a=(1,1,1)$ and $b=(2,1,1)$.

Proposition 2.8. Let $(R, \mathfrak{m}, k)$ be a complete Gorenstein local domain of Krull dimension 3. Suppose that, in addition, $R \supset k, k$ is infinite, and $e(R) \leq 3$. Let $\left(x_{1}, x_{2}, x_{3}\right) R$ be a minimal reduction of $\mathfrak{m}$. Then there is a minimal prime over $I=\left(x_{1}^{3}-x_{2} x_{3}, x_{2}^{2}-x_{1} x_{3}, x_{3}^{2}-x_{1}^{2} x_{2}\right)$ which is not Gorenstein. In particular, $R$ is not Shimoda. 
Let us give now a proof of Theorem 2.3 using Propositions 2.6 and 2.8.

Proof of Theorem 2.3 using Propositions 2.6 and 2.8. Let $R$ be a Shimoda ring of Krull dimension $d \geq 2$ as in Theorem 2.3. By Remark 2.4, one can suppose that $2 \leq d \leq 3$. If $R$ is regular, by Proposition $2.6, d \neq 3$. Suppose now that $R$ is complete, $R \supset k, k$ is infinite, and $e(R) \leq 3$. By Remark 2.2, $R$ is Cohen-Macaulay, and since $R$ is complete, it admits a canonical module (see, e.g., [BH, Corollary 3.3.8]). By Remark 2.2 again, $R$ is a UFD. Hence, $R$ is Gorenstein (see, e.g., [BH, Corollary 3.3.19]). Therefore, by Proposition 2.8, $d \neq 3$.

Before proving Proposition 2.6, we note the following reasonably elementary fact.

Lemma 2.9. Let $(S, \mathfrak{n})$ be a Cohen-Macaulay local ring of Krull dimension 2. Let $x, y$ be a system of parameters of $S$. Let $p, q, r, s \in \mathbb{N}$, with $1 \leq r<p$ and $1 \leq s<q$. Then

$$
\operatorname{length}_{S}\left(S /\left(x^{p}, y^{q}, x^{r} y^{s}\right)\right)=[p q-(p-r)(q-s)] \cdot \operatorname{length}_{S}(S /(x, y)) .
$$

Proof. Let $I=(x, y)$. Since $I$ is generated by a regular sequence, there is a natural graded isomorphism $(S / I)[X, Y] \cong \mathbf{G}(I)$, sending $X$ to $x+I^{2}$ and $Y$ to $y+I^{2}$, between the polynomial ring in two indeterminates $X, Y$ over $S / I$ and the associated graded ring $G=\mathbf{G}(I)=\bigoplus_{n \geq 0} I^{n} / I^{n+1}$ of $I$. If $z \in S$, let $z^{*}$ denote its initial form in $G$; that is, $z^{*}=z+I^{m}$, where $z \in I^{m-1} \backslash I^{m}$, and $0^{*}=0$. In particular, $\left(x^{*}\right)^{n}=\left(x^{n}\right)^{*}$ and $\left(y^{*}\right)^{m}=\left(y^{m}\right)^{*}$, for all $n, m \geq 1$. If $J$ is an ideal of $S$, let $J^{*}$ denote the homogeneous ideal of $G$ generated by all the initial forms of elements of $J$. One has $J_{n}^{*}=\left(J \cap I^{n}\right)+I^{n+1} / I^{n+1}$ for $n \geq 0$ (see, e.g., [VV, p. 94]).

Now take $J=\left(x^{p}, y^{q}, x^{r} y^{s}\right) \subset I$. Observe that, for $n \geq p+q, I^{n} \subseteq J$ and $\left(J^{*}\right)_{n}=G_{n}$. For each $n \geq 0$, consider the short exact sequences of $S$-modules:

$$
0 \rightarrow\left(J+I^{n+1}\right) / J \rightarrow\left(J+I^{n}\right) / J \rightarrow\left(J+I^{n}\right) /\left(J+I^{n+1}\right) \rightarrow 0 .
$$

Hence, $\operatorname{length}_{S}(S / J)=\sum_{n=0}^{p+q-1} \operatorname{length}_{S}\left(\left(J+I^{n}\right) /\left(J+I^{n+1}\right)\right)$. But

$$
\frac{J+I^{n}}{J+I^{n+1}} \cong \frac{I^{n}}{I^{n} \cap\left(J+I^{n+1}\right)} \cong \frac{I^{n}}{\left(J \cap I^{n}\right)+I^{n+1}} \cong \frac{G_{n}}{J_{n}^{*}} .
$$


Thus,

$$
\begin{aligned}
\operatorname{length}_{S}(S / J) & =\sum_{n=0}^{p+q-1} \operatorname{length}_{S}\left(G_{n} / J_{n}^{*}\right)=\sum_{n \geq 0} \operatorname{length}_{S}\left(G_{n} / J_{n}^{*}\right) \\
& =\operatorname{length}_{S}\left(G / J^{*}\right) .
\end{aligned}
$$

Let $L \subseteq J^{*}$ be the ideal generated by the initial forms of $x^{p}, y^{q}$, and $x^{r} y^{s}$ in $G$. Since the regular sequence $x, y$ is permutable, by [KS, Remark 4(4)], $L=J^{*}$. Hence, $\operatorname{length}_{S}\left(G / J^{*}\right)=\operatorname{length}_{S}(G / L)<\infty$. Through the isomorphism $(S / I)[X, Y] \cong \mathbf{G}(I)$, one sees that $G / L$ is isomorphic to the free $S / I$-module with basis $X^{i} Y^{j}$, where $(i, j) \in\{0, \ldots, p-1\} \times\{0, \ldots, s-1\}$ or $(i, j) \in\{0, \ldots, r-1\} \times\{s, \ldots, q-1\}$. Therefore, length $_{S}(G / L)=[p q-(p-r)$ $(q-s)] \cdot$ length $_{S}(S / I)$.

REMARK 2.10. The result of Kiyek and Stückrad (see [KS, Remark 4(4)]) that we have used in the proof of Lemma 2.9, which deals only with the case where the regular sequence at hand is permutable, says precisely that $x^{p}, y^{q}, x^{r} y^{s}$ is an $I$-standard base of $J=\left(x^{p}, y^{q}, x^{r} y^{s}\right)$ (see, e.g., [HIO, Section 13]). In this respect, one can prove the following fact, which uses a generalization of a classic theorem of Rees (see [BH, Theorem 1.1.7]).

Theorem 2.11. Let $R$ be a commutative ring, and let $\underline{z}=z_{1}, \ldots, z_{n}$ be an $R$-sequence. Set $I=\left(z_{1}, \ldots, z_{n}\right)$. Let $\underline{Z}=Z_{1}, \ldots, Z_{n}$ be indeterminates over $R$.

(a) If $F(\underline{Z}) \in R[\underline{Z}]$ is homogeneous of degree $d$ and $F(\underline{z}) \in I^{d+i}$, then $F(\underline{Z}) \in I^{i}[\underline{Z}]$.

(b) If $m_{1}, \ldots, m_{s}$ is a finite set of monomials in $\underline{z}$, then $m_{1}, \ldots, m_{s}$ is an $I$-standard base of $J=\left(m_{1}, \ldots, m_{s}\right)$.

Proof. If $i=0$, the result is trivial. Rees's theorem is the case $i=1$. Suppose that $i>1$ and that we have established the result in the case where $i$ is replaced by $i-1$. Suppose then that $F(\underline{Z}) \in R[\underline{Z}]$ is homogeneous of degree $d$ and that $F(\underline{z}) \in I^{d+i}$. Then $F(\underline{z}) \in I^{d+i-1}$, so by the inductive hypothesis, the coefficients of $F$ lie in $I^{i-1}$. Thus, with $\lambda=\left(\lambda_{1}, \ldots, \lambda_{n}\right)$,

$$
F(\underline{Z})=\sum_{\lambda_{1}+\cdots+\lambda_{n}=d} a_{\lambda} Z_{1}^{\lambda_{1}} \cdots Z_{n}^{\lambda_{n}},
$$

for $a_{\lambda} \in I^{i-1}$. Hence, for each such $\lambda$,

$$
a_{\lambda}=\sum_{\mu_{1}+\cdots+\mu_{n}=i-1} b_{\lambda, \mu} z_{1}^{\mu_{1}} \cdots z_{n}^{\mu_{n}},
$$


for elements $b_{\lambda, \mu}$ in $R$, with $\mu=\left(\mu_{1}, \ldots, \mu_{n}\right)$ and $\mu_{1}+\cdots+\mu_{n}=i-1$. Let $\xi=\left(\xi_{1}, \ldots, \xi_{n}\right)=\lambda+\mu$, so that $\xi_{1}+\cdots+\xi_{n}=d+i-1$, and let

$$
H(\underline{Z})=\sum_{\left(\lambda_{1}+\cdots+\lambda_{n}=d\right)} \sum_{\left(\mu_{1}+\cdots+\mu_{n}=i-1\right)} b_{\lambda, \mu} Z_{1}^{\xi_{1}} \cdots Z_{n}^{\xi_{n}} \in R[\underline{Z}] .
$$

Then $H$ is homogeneous of degree $d+i-1$ and $H(\underline{z})=F(\underline{z}) \in I^{d+i}$. By Rees's theorem, the coefficients $b_{\lambda, \mu}$ of $H$ lie in $I$, and it follows that each $a_{\lambda}$ lies in $I^{i}$, which proves (a). Let $m_{1}, \ldots, m_{s}$ be monomials in $\underline{z}$, where $m_{i}=z_{1}^{\alpha_{1, i}} \cdots z_{n}^{\alpha_{n, i}}$, for $i=1, \ldots, s$. Let $\alpha_{i}=\alpha_{1, i}+\cdots+\alpha_{n, i}$ be the degree of $m_{i}$, which is well defined because of the standard isomorphism $(R / I R)\left[Z_{1}, \ldots, Z_{n}\right] \rightarrow \mathbf{G}(I)=\bigoplus_{d \geq 0} I^{d} / I^{d+1}$, sending $Z_{i}$ to $z_{i}+I^{2}$. Let $\delta_{1}<$ $\cdots<\delta_{t}$ be the degrees occurring in $\left\{\alpha_{1}, \ldots, \alpha_{s}\right\}, t \geq 1$. Let $J_{i}$ denote the ideal of $R$ generated by those monomials among $m_{1}, \ldots, m_{s}$ that have degree $\delta_{i}$. We must show that, for $d \geq 0$,

$$
J \cap I^{d}=m_{1} I^{d-\alpha_{1}}+\cdots+m_{s} I^{d-\alpha_{s}},
$$

or equivalently,

$$
J \cap I^{d}=J_{1} I^{d-\delta_{1}}+\cdots+J_{t} I^{d-\delta_{t}}
$$

understanding that $I^{r}=R$ whenever $r \leq 0$ and noting that $I$ and $J$ are not comaximal (see [VV, p. 94, lines 23-24]). We prove (1) by induction on $t \geq 1$.

Item (a) easily yields the case where $t=1$, that is, where all the monomials have the same degree. So we suppose that $t \geq 2$ and that (1) holds when $t$ is replaced by $t-1$. Suppose first of all that $d \leq \delta_{t}$. Then, by induction,

$$
\begin{aligned}
J \cap I^{d} & =\left(J_{1}+\cdots+J_{t}\right) \cap I^{d}=\left(\left(J_{1}+\cdots+J_{t-1}\right) \cap I^{d}\right)+J_{t} \\
& =J_{1} I^{d-\delta_{1}}+\cdots+J_{t-1} I^{d-\delta_{t-1}}+J_{t} I^{d-\delta_{t}},
\end{aligned}
$$

since $I^{d-\delta_{t}}=R$ in this case. Finally, suppose on the other hand that $d>\delta_{t}$. Then

$$
J \cap I^{d}=J \cap I^{\delta_{t}} \cap I^{d}=\left(J_{1}+\cdots+J_{t}\right) \cap I^{\delta_{t}} \cap I^{d}=\left(J_{1} I^{\delta_{t}-\delta_{1}}+\cdots+J_{t}\right) \cap I^{d},
$$

by the case just considered. But the ideal $J_{1} I^{\delta_{t}-\delta_{1}}+\cdots+J_{t}$ is generated by monomials in $\underline{z}$ of degree $\delta_{t}$, so by the case $t=1$,

$$
\left(J_{1} I^{\delta_{t}-\delta_{1}}+\cdots+J_{t}\right) \cap I^{d}=\left(J_{1} I^{\delta_{t}-\delta_{1}}+\cdots+J_{t}\right) I^{d-\delta_{t}}=J_{1} I^{d-\delta_{1}}+\cdots+J_{t} I^{d-\delta_{t}},
$$

as required. 
To prove Proposition 2.6, we are going to use formulas from the theory of multiplicities (see, e.g., [BH, Chapter 4, Section 6] and [HS, Chapter 11, Sections 1 and 2]).

Proof of Proposition 2.6. Let $\widehat{R}$ be the completion of $R$. Since $R$ is regular and local with maximal ideal $\mathfrak{m}$ generated by the regular system of parameters $x_{1}, x_{2}, x_{3}$, then $\widehat{R}$ is a regular local ring with maximal ideal $\mathfrak{m} \widehat{R}=\left(x_{1}, x_{2}, x_{3}\right) \widehat{R}$, generated by the regular system of parameters $x_{1}, x_{2}, x_{3}$ (considered in $\widehat{R})$. Let $I=\left(v_{1}, v_{2}, D\right)$ be the HN ideal associated to $x_{1}, x_{2}, x_{3}$ and $a, b \in \mathbb{N}^{3}$ in $R$. Then $I \widehat{R}=\left(v_{1}, v_{2}, D\right) \widehat{R}$ is the HN ideal associated to $x_{1}, x_{2}, x_{3}$ and $a, b \in \mathbb{N}^{3}$, regarded in $\widehat{R}$. Since $I=I \widehat{R} \cap R$, if we prove that $I \widehat{R}$ is prime, then $I$ will be prime, too. Therefore we can suppose that $R$ is complete.

Until further notice, we assume only that $R$ is a complete Cohen-Macaulay local ring of dimension 3 and that $x_{1}, x_{2}, x_{3}$ generates a reduction of $\mathfrak{m}$. We do this so that we can avoid repeating core aspects of the argument when we prove Proposition 2.8.

Take $\mathfrak{p}$ any associated prime of $I$, hence of height 2 . Let $D=R / \mathfrak{p}$. Then $D$ is a 1-dimensional Noetherian domain. Let $V$ be its integral closure in its quotient field $K$. Since $R$ is a complete local ring, $R$ is a Nagata ring (see, e.g., [Ma1, p. 234]), and hence $V$ is a finite $D$-module and, in particular, $V$ is Noetherian as a ring. Since $D$ is a Noetherian complete local domain, its integral closure $V$ is a local ring (see, e.g., [Di, Corollary 6.1, p. 116]). Therefore, $V$ is a 1-dimensional Noetherian local integrally closed ring, hence a discrete valuation ring (DVR).

Let $\nu$ denote the corresponding valuation on $K$. By abuse of notation, let $x_{i}$ denote the image in $V$ of each $x_{i}$, and set $\nu_{i}=\nu\left(x_{i}\right)$. In $V, x_{1}^{c_{1}}=x_{2}^{b_{2}} x_{3}^{a_{3}}$, $x_{2}^{c_{2}}=x_{1}^{a_{1}} x_{3}^{b_{3}}$, and $x_{3}^{c_{3}}=x_{1}^{b_{1}} x_{2}^{a_{2}}$. Applying $\nu$ to these equalities, one gets the following system of equations:

$$
\left.\begin{array}{l}
c_{1} \nu_{1}=b_{2} \nu_{2}+a_{3} \nu_{3} \\
c_{2} \nu_{2}=a_{1} \nu_{1}+b_{3} \nu_{3} \\
c_{3} \nu_{3}=b_{1} \nu_{1}+a_{2} \nu_{2}
\end{array}\right\},
$$

the third equation (say) being the sum of the first two. So we reduce to a system of two linearly independent equations, considered over $\mathbb{Q}$, whose solution is $\left(\nu_{1}, \nu_{2}, \nu_{3}\right)=q \cdot m(a, b)$, for some $q \in \mathbb{Q}, q=u / v, u, v \in \mathbb{N}$ (see [OP, Remark 4.4]). 
Set $l=\operatorname{gcd}\left(\nu_{1}, \nu_{2}, \nu_{3}\right) \in \mathbb{N}$. Since $v\left(\nu_{1}, \nu_{2}, \nu_{3}\right)=u\left(m_{1}, m_{2}, m_{3}\right)$ and $\operatorname{gcd}\left(m_{1}\right.$, $\left.m_{2}, m_{3}\right)=1$, then $v l=u$ and $l=q \in \mathbb{N}$. Hence, $\nu_{i}=l m_{i}$.

Clearly, $x_{1} R+I=\left(x_{1}, x_{2}^{c_{2}}, x_{3}^{c_{3}}, x_{2}^{b_{2}} x_{3}^{a_{3}}\right)$ is an $\mathfrak{m}$-primary ideal of $R$. Set $S=$ $R / x_{1} R$, and (by abuse of notation) consider $x_{2}, x_{3}$ as a regular sequence in $S$ and a system of parameters of $S$. Since $R /\left(x_{1} R+I\right) \cong S /\left(x_{2}^{c_{2}}, x_{3}^{c_{3}}, x_{2}^{b_{2}} x_{3}^{a_{3}}\right) S$ (and $\left.x_{1} R \subset \operatorname{Ann}_{R}(S)\right)$, then $\operatorname{length}_{R}\left(R /\left(x_{1} R+I\right)\right)=\operatorname{length}_{S}\left(S /\left(x_{2}^{c_{2}}, x_{3}^{c_{3}}\right.\right.$, $\left.\left.x_{2}^{b_{2}} x_{3}^{a_{3}}\right) S\right)$, which by Lemma 2.9 is equal to $m_{1} \cdot \operatorname{length}_{S}\left(S /\left(x_{2}, x_{3}\right) S\right)$. But $S /\left(x_{2}, x_{3}\right) S \cong R /\left(x_{1}, x_{2}, x_{3}\right)$, so

$$
\operatorname{length}_{S}\left(S /\left(x_{2}, x_{3}\right) S\right)=\operatorname{length}_{R}\left(R /\left(x_{1}, x_{2}, x_{3}\right)\right)=e_{R}\left(x_{1}, x_{2}, x_{3}\right),
$$

because $R$ is Cohen-Macaulay of Krull dimension $d=3$ and $\left(x_{1}, x_{2}, x_{3}\right)$ is an $\mathfrak{m}$-primary ideal [HS, Proposition 11.1.10]. Moreover, as $\left(x_{1}, x_{2}, x_{3}\right)$ is a reduction of $\mathfrak{m}$, then $e_{R}\left(x_{1}, x_{2}, x_{3}\right)=e(R)$ [HS, Corollary 1.2.5 and Proposition 11.2.1].

Since $x_{1} R+I \subseteq x_{1} R+\mathfrak{p}$, then $\operatorname{length}_{R}\left(R /\left(x_{1} R+I\right)\right) \geq \operatorname{length}_{R}(R /$ $\left.\left(x_{1} R+\mathfrak{p}\right)\right)$. But

$\operatorname{length}_{R}\left(R /\left(x_{1} R+\mathfrak{p}\right)\right)=\operatorname{length}_{R / \mathfrak{p}}\left((R / \mathfrak{p}) /\left(x_{1} \cdot R / \mathfrak{p}\right)\right)=\operatorname{length}_{D}\left(D / x_{1} D\right)$

Observe that $x_{2}^{c_{2}}, x_{3}^{c_{3}} \in x_{1} D$, and so $x_{1} D$ is an $\mathfrak{m} / \mathfrak{p}$-primary ideal of the 1 dimensional Cohen-Macaulay local domain $(D, \mathfrak{m} / \mathfrak{p}, k)$. By [HS, Proposition 11.1.10], length ${ }_{D}\left(D / x_{1} D\right)=e_{D}\left(x_{1} ; D\right)$.

On the other hand, $V$ is a finite Cohen-Macaulay $D$-module of $\operatorname{rank}_{D}(V)=1$, and so $e_{D}\left(x_{1} ; D\right)=e_{D}\left(x_{1} ; D\right) \cdot \operatorname{rank}_{D}(V)=\operatorname{length}_{D}\left(V / x_{1} V\right)$ [BH, Corollary 4.6.11].

Set $r=\left[k_{V}: k_{D}\right]$, the degree of the extension of the residue fields of $V$ and of $D$. Then length $D\left(V / x_{1} V\right)=r \cdot \operatorname{length}_{V}\left(V / x_{1} V\right)$. Finally, since $V$ is a DVR, length $V\left(V / x_{1} V\right)=\nu\left(x_{1}\right)=\nu_{1}=l m_{1}$.

Therefore, putting together all the (in)equalities, we have

$$
m_{1} \cdot e(R)=\operatorname{length}_{R}\left(R /\left(x_{1} R+I\right)\right) \geq \operatorname{length}_{R}\left(R /\left(x_{1} R+\mathfrak{p}\right)\right)=r m_{1} .
$$

Observe that in all this reasoning, we have only used the facts that $R$ is a complete Cohen-Macaulay local ring of dimension 3 and that $x_{1}, x_{2}, x_{3}$ generates a reduction of $\mathfrak{m}$.

Now, using the fact that $R$ is regular, we have $e(R)=1$, and we deduce from (2) that

$$
\operatorname{length}_{R}\left(R /\left(x_{1} R+I\right)\right)=\operatorname{length}_{R}\left(R /\left(x_{1} R+\mathfrak{p}\right)\right) .
$$


On tensoring the exact sequence $0 \rightarrow \mathfrak{p} / I \rightarrow R / I \rightarrow R / \mathfrak{p} \rightarrow 0$ by $R / x_{1} R$, one obtains the exact sequence

$$
0 \rightarrow L / x_{1} L \rightarrow R /\left(x_{1} R+I\right) \rightarrow R /\left(x_{1} R+\mathfrak{p}\right) \rightarrow 0
$$

where $L=\mathfrak{p} / I$, because $x_{1}$ is not in $\mathfrak{p}$, so $x_{1} R \cap \mathfrak{p}=x_{1} \mathfrak{p}$. Then, from the equality length $_{R}\left(R /\left(x_{1} R+I\right)\right)=\operatorname{length}_{R}\left(R /\left(x_{1} R+\mathfrak{p}\right)\right)$, one deduces that length $_{R}\left(L / x_{1} L\right)=0$, and hence that $L=x_{1} L$, which by Nakayama's lemma implies that $L=0$ and $I=\mathfrak{p}$.

Now we turn to the proof of Proposition 2.8.

Proof of Proposition 2.8. First observe that since $k$ is infinite, there exist $x_{1}, x_{2}, x_{3}$ in $\mathfrak{m}$ such that $\left(x_{1}, x_{2}, x_{3}\right)$ is a minimal reduction of $\mathfrak{m}$. In particular, $x_{1}, x_{2}, x_{3}$ is a system of parameters in $R$. Since $(R, \mathfrak{m}, k)$ is a complete Noetherian local domain and since $R \supset k$, there exists a $k$-algebra homomorphism $\varphi: k\left[\left[X_{1}, X_{2}, X_{3}\right]\right] \rightarrow R$, from the power series ring in the three indeterminates $X_{1}, X_{2}, X_{3}$ over $k$ to $R$, with $\varphi\left(X_{i}\right)=x_{i}$, and such that if $S=\operatorname{im}(\varphi)=k\left[\left[x_{1}, x_{2}, x_{3}\right]\right]$, then $S \cong k\left[\left[X_{1}, X_{2}, X_{3}\right]\right]$ via $\varphi, S$ is a complete regular local ring, and $R$ is a finite extension of $S$ (see, e.g., [Ma2, Theorem 29.4 and its proof $])$. Let $\psi: k\left[\left[X_{1}, X_{2}, X_{3}\right]\right] \rightarrow k[[t]]$ be defined as $\psi\left(X_{1}\right)=t^{3}, \psi\left(X_{2}\right)=t^{4}$, and $\psi\left(X_{3}\right)=t^{5}$, where $t$ is an indeterminate over $k$. Then $\operatorname{ker}(\psi)=\left(X_{1}^{3}-X_{2} X_{3}, X_{2}^{2}-X_{1} X_{3}, X_{3}^{2}-X_{1}^{2} X_{2}\right)$ (see, e.g., [OP, Theorem 7.8], where a proof for the polynomial case is given). Set $J=\varphi(\operatorname{ker}(\psi))$, which is a prime ideal of $S$, and set $I=J R=\left(x_{1}^{3}-x_{2} x_{3}, x_{2}^{2}-\right.$ $\left.x_{1} x_{3}, x_{3}^{2}-x_{1}^{2} x_{2}\right)$, which is the HN ideal of $R$ associated to $x_{1}, x_{2}, x_{3}$, and set $a=(1,1,1), b=(2,1,1)$.

Take now any minimal prime $\mathfrak{p}$ over $I$, and suppose that $\mathfrak{p}$ is Gorenstein. We will reach a contradiction.

Since $J R=I \subseteq \mathfrak{p}$ and since $S \subset R$ is an integral extension, then $J \subseteq$ $I \cap S \subseteq \mathfrak{p} \cap S$, and $S /(\mathfrak{p} \cap S) \subset R / \mathfrak{p}$ is an integral extension. Hence, $\mathfrak{p} \cap S$ is a prime ideal of $S$ of height 2 and $J=\mathfrak{p} \cap S$. Set $A=S / J \cong k\left[\left[t^{3}, t^{4}, t^{5}\right]\right] \subset k[[t]]$ and $D=R / \mathfrak{p}$. By the Auslander-Buchsbaum formula, $R$ is a free $S$-module of $\operatorname{rank}_{S}(R)=e(S) \cdot \operatorname{rank}_{S}(R)=e(R)=$ : e (see, e.g., [BH, Corollary 4.6.9]). By base change, it follows that $R / I$ is a free $A$-module of rank $e$. Hence, $D$ is a torsion-free $A$-module of rank $e^{\prime}$, where $1 \leq e^{\prime} \leq e$.

We claim that $e^{\prime}>1$. Indeed, suppose that $e^{\prime}=1$. Then the quotient field of $D$ can be identified with the quotient field of $A$. By [E, Exercise 21.11], the quotient field of $A$ is $k((t))$ and the integral closure $V$ of $A$ is $k[[t]]$. Since $A$ and $D$ have the same quotient field with $A \subset D$ a finite and hence integral 
extension, $V$ is also the integral closure of $D$. By [E, Exercise 21.11], the conductor $A:_{A} V$ of $V$ into $A$ equals $\left(t^{3}, t^{4}, t^{5}\right) A=\mathfrak{m}_{A}$, the maximal ideal of $A$. Since $A:_{A} V$ is also an ideal in $V$ and since $t^{3} \in A:_{A} V$, we therefore have

$$
t^{3} V \subseteq A:_{A} V=\mathfrak{m}_{A}=\left(t^{3}, t^{4}, t^{5}\right) A \subseteq\left(t^{3}, t^{4}, t^{5}\right) V=t^{3} V .
$$

Therefore, $t^{3} V=A:_{A} V=\mathfrak{m}_{A}$. Now $x_{1} D$ is a reduction of the maximal ideal $\mathfrak{m}_{D}$ of $D$, since $\left(x_{1}, x_{2}, x_{3}\right) R$ is a reduction of $\mathfrak{m}$. Hence, the integral closure $\overline{x_{1} D}$ of the ideal $x_{1} D$ equals $\mathfrak{m}_{D}$, so $x_{1} V=\overline{\left(x_{1} D\right)} V=\mathfrak{m}_{D} V$ (see, e.g., [HS, Proposition 6.8.1]). Since $x_{1} V=t^{3} V$ and $A \subset D$ is integral, it follows that

$$
\mathfrak{m}_{D} \subseteq \mathfrak{m}_{D} V=t^{3} V=\mathfrak{m}_{A} \subseteq \mathfrak{m}_{D}
$$

Hence, $\mathfrak{m}_{D}=\mathfrak{m}_{D} V=t^{3} V$. In particular, $D \neq V$ and $\mathfrak{m}_{D}$ is also an ideal of $V$. Hence, $\mathfrak{m}_{D} \subseteq D:_{D} V \subsetneq D$, so $\mathfrak{m}_{D}=D:_{D} V=t^{3} V$. Using the fact that $D$ is Gorenstein and [HS, Theorem 12.2.2], one has

$$
\begin{aligned}
2 & =2 \cdot \operatorname{length}_{D}\left(D / \mathfrak{m}_{D}\right)=2 \cdot \operatorname{length}_{D}\left(D /\left(D:_{D} V\right)\right) \\
& =\operatorname{length}_{D}\left(V /\left(D:_{D} V\right)\right)=\operatorname{length}_{D}\left(V / t^{3} V\right)=r \cdot \operatorname{length}_{V}\left(V / t^{3} V\right)=3 r,
\end{aligned}
$$

where $r=\left[k_{V}: k\right]$, which is a contradiction. Thus, $e^{\prime} \geq 2$.

Now, $R / I$ and $A$ are local rings with the same residue field. Hence, since $x_{1} A$ is an $\mathfrak{m}_{A}$-primary ideal of the Noetherian local domain $A$ and $\operatorname{rank}_{A}(D)=e^{\prime}$, by [HS, Corollary 11.2.6], and regarding $D$ as an $R / I$ module, we have the following:

$$
e_{R / I}\left(x_{1} \cdot R / I ; D\right)=e_{A}\left(x_{1} A ; D\right)=e_{A}\left(x_{1} A ; A\right) \cdot \operatorname{rank}_{A}(D)=3 e^{\prime} \geq 6 .
$$

Analogously, since $\operatorname{rank}_{A}(R / I)=e \leq 3$ by hypothesis, then

$$
e_{R / I}\left(x_{1} \cdot R / I ; R / I\right)=e_{A}\left(x_{1} A ; R / I\right)=e_{A}\left(x_{1} A ; A\right) \cdot \operatorname{rank}_{A}(R / I)=3 e \leq 9 .
$$

But, by the associativity formula (see, e.g., [HS, Theorem 11.2.4]), letting $\mathfrak{p}$ vary through the minimal primes in $R$ over $I$, we have

$$
\begin{aligned}
9 & \geq e_{R / I}\left(x_{1} ; R / I\right)=\sum_{\mathfrak{p}} e_{R / I}\left(x_{1} ; R / \mathfrak{p}\right) \cdot \operatorname{length}_{R_{\mathfrak{p}}}\left(R_{\mathfrak{p}} / I R_{\mathfrak{p}}\right) \\
& \geq 6 \cdot(\text { the number of such } \mathfrak{p}) .
\end{aligned}
$$

Hence, the number of such $\mathfrak{p}$ equals 1 , and for this unique $\mathfrak{p}, \operatorname{length}_{R_{\mathfrak{p}}}\left(R_{\mathfrak{p}} /\right.$ $\left.I R_{\mathfrak{p}}\right)=1$. Therefore, $I=\mathfrak{p}$, which is a contradiction, since $I$ is not Gorenstein and $\mathfrak{p}$ is Gorenstein. 


\section{$\S 3$. The Shimoda conjecture in the setting of a standard graded algebra}

In connection with the Shimoda property for affine rings, we note the following result.

Proposition 3.1. Let $S$ be an affine domain of Krull dimension $d$ at least 3. Then $S$ contains a prime ideal $P$ that requires more than height $(P)$ generators.

Proof. Since $S$ is an excellent domain, the regular $\operatorname{locus} \operatorname{Reg}(S)$ is a nonempty Zariski-open subset of $\operatorname{Spec}(S)$. Hence, there exists a nonzero element $s \in S$ such that $S_{s}$ is a regular ring. Since $S$ is a Hilbert ring with (0) a prime ideal, there exists a maximal ideal $M \in S$ such that $s \notin M$, so $S_{M}$ is a regular local ring. Now height $(M)=d \geq 3$, and so, by Theorem 2.3 (a) and its proof, there exists $\mathfrak{p} \in \operatorname{Spec}\left(S_{M}\right)$ having dimension 1 such that $\mu(\mathfrak{p})=d$. Set $P=\mathfrak{p} \cap S$. Since $\mathfrak{p}=P_{M}$, it follows that height $(P)=d-1$, yet $P$ requires at least $d$ generators.

REMARK 3.2. Note, however, that if $S$ is also a standard graded algebra, the preceding result does not provide any information as to whether the resulting prime ideal $P$ is or is not homogeneous.

We now consider a setup different but analogous to the local case considered in Section 2, influenced by the well-known similarities between the theories of local and standard graded rings. So let $A=k\left[x_{1}, \ldots, x_{n}\right]$ be a standard graded algebra over the field $k$; that is, $A$ is graded by the nonnegative integers $\mathbb{N}_{0}$ with $k$ sitting in degree 0 and each of the $x_{i}$ having degree 1. For simplicity, we suppose that $k$ is infinite (in fact, eventually we will suppose that $k$ has characteristic 0 or is algebraically closed). Let $\mathcal{M}:=\left(x_{1}, \ldots, x_{n}\right) A$ denote the irrelevant ideal of $A$, and we take $n$ to be the minimal number of homogeneous generators $\mu(\mathcal{M})$ of $\mathcal{M}$ (see [BH, Proposition 1.5.15(a)], noting that $\mu(\mathcal{M})=\mu\left(\mathcal{M}_{\mathcal{M}}\right)$ ). We fix this notation. We also suppose that $A$ is a Shimoda ring in the graded sense, or a gr-Shimoda ring for short, meaning that each relevant homogeneous prime ideal $\mathfrak{p}$ of $A$ is generated by height $(\mathfrak{p})$ elements, that is, is of the principal class; note that these generators can be chosen to be homogeneous (once again, see $[\mathrm{BH}$, Proposition 1.5.15(a)]).

Finally, we suppose throughout that $A$ has Krull dimension at least 2. 
Proposition 3.3. Suppose that the gr-Shimoda ring A has Krull dimension $d$ (with $d \geq 2$ ). Then $A$ is a Gorenstein domain that satisfies the Serre condition $\left(R_{d-1}\right)$.

Proof. On taking $\mathfrak{p}$ to be a minimal prime (necessarily homogeneous) in the above condition, we see that $\mathfrak{p}=(0)$, so that $A$ is a domain. Next, let $\mathfrak{p}$ be a homogeneous prime that is maximal with respect to the property of being relevant. By Davis's result [D, Remark p. 203], $\mathfrak{p}$ is generated by a regular sequence of length height( $(\mathfrak{p})$. It follows that $\mathcal{M}$ contains a regular sequence of length height $(\mathcal{M})$. Hence, $A$ is Cohen-Macaulay (see [BH, Exercise 2.1.27(c)]).

Next, since $A$ is a homomorphic image of a polynomial ring (with the standard grading) by a homogeneous morphism of degree $0, A$ has a graded canonical module $C$ (see [BH, Example 3.6.10 and Proposition 3.6.12(b)]). By [E, Exercise 21.18(b)], we may take $C$ to be a homogeneous ideal of $A$. If $C=A$, then $A$ is Gorenstein [BH, Proposition 3.6.11]. Suppose, on the other hand, that $C$ is a proper ideal in $A$. By [BH, Propositions 3.6.9 and 3.3.18], $C$ is an unmixed ideal of height 1 whose (necessarily homogeneous) associated primes are therefore principal, by the gr-Shimoda property. Let $\mathfrak{p}$ be any one of these associated primes, with $\mathfrak{p}=(p)$, say. Then each $A_{\mathfrak{p}}$ is a DVR, so that each localization $C_{\mathfrak{p}}$ is a principal ideal, say, $C_{\mathfrak{p}}=p^{t_{p}} A_{\mathfrak{p}}$. It is easily seen that $C=\left(\prod_{p} p^{t_{p}}\right) A$, since the latter ideal has the same associated primes as $C$ and agrees with $C$ locally at each of these primes. Hence, $C$ is principal. That $A$ is Gorenstein now follows from [BH, Proposition 3.6.11].

Finally, to show that $A$ satisfies $\left(R_{d-1}\right)$, we examine $A_{\mathfrak{p}}$ for each prime ideal $\mathfrak{p}$ of height $h$, with $h \leq d-1$. Clearly, we need only consider the case where $\mathfrak{p}$ is nonhomogeneous, so that $h>0$. By [BH, Theorem 1.5.8, first paragraph of proof], or by [F, (5.1) Lemma, (b)], we see that for such $\mathfrak{p}, A_{\mathfrak{p}}$ is a regular local ring, and the result follows.

We next consider the effect of Noether normalization applied to $A$, noting that $k$ is infinite. This result highlights another way that the present situation is to an extent analogous to that of Section 2.

We distinguish between the Krull dimension of $A$, denoted $\mathrm{k}-\operatorname{dim}(A)$, and the dimension of $A$ as a projective scheme, denoted $\operatorname{pr}-\operatorname{dim}(A)$. Thus, $\mathrm{k}-\operatorname{dim}(A)=\operatorname{pr}-\operatorname{dim}(A)+1$ (see [E, pp. 286-287]).

Proposition 3.4. Let $d=\mathrm{k}-\operatorname{dim}(A)$. Then there exists a regular sequence of homogeneous elements of degree 1 in $A$, which we relabel as $x_{1}, \ldots, x_{d}$, 
such that $A=k\left[x_{1}, \ldots, x_{n}\right]$ with $A$ a free finitely generated graded module over the subring $B:=k\left[x_{1}, \ldots, x_{d}\right], B$ being isomorphic to the polynomial ring over $k$ in $d$ variables (with the standard grading) under the natural homogeneous mapping of degree 0.

Proof. See [BH, Theorem 1.5.17(c)], together with the discussion in [St, Section 3, especially p. 63]. Note also that $n$ remains invariant under this rewriting of $A$, as $n=\mu(\mathcal{M})=\mu\left(\mathcal{M}_{\mathcal{M}}\right)$.

We now show how to reduce dimension for gr-Shimoda rings.

Proposition 3.5. Let a be a homogeneous element of the gr-Shimoda ring $A$, a lying in $\mathcal{M} \backslash \mathcal{M}^{2}$. Then $A / a A$ is again a gr-Shimoda ring.

Proof. First of all, we note that such an element $a$ exists. By the graded version of Nakayama's lemma, $\mathcal{M} \neq \mathcal{M}^{2}$ since $A$ is not a field. Since $\mathcal{M}$ is a homogeneous ideal, it has a set of homogeneous generators not all of which can lie in $\mathcal{M}^{2}$, by the previous observation. Pick $a$ to be a suitable member of this set of generators.

Clearly, $A / a A$ is a standard graded $\operatorname{ring}($ with $\mathrm{k}-\operatorname{dim}(A / a A)$ at least 1 ) having irrelevant ideal $\mathcal{M} / a A$. Consider a relevant homogeneous prime ideal $\mathfrak{p}$ of $A / a A$. Then $\mathfrak{p}=\mathfrak{P} / a A$ for a unique relevant homogeneous prime ideal $\mathfrak{P}$ of $A$ that contains $a$. Since $a \in \mathcal{M} \backslash \mathcal{M}^{2}$, a fortiori, $a \in \mathfrak{P} \backslash \mathcal{M}$. Hence, $a$ is a minimal homogeneous generator of $\mathfrak{P}$, so that

$$
\mu(\mathfrak{p})=\mu(\mathfrak{P})-1=\operatorname{height}(\mathfrak{P})-1=\operatorname{height}(\mathfrak{p}),
$$

and the result follows.

We can now give our main result.

TheOREM 3.6. Suppose that in the gr-Shimoda ring A, the base field $k$ has characteristic 0 . Then $\operatorname{pr}-\operatorname{dim}(A) \leq 2$.

Proof. We suppose that $\operatorname{pr}-\operatorname{dim}(A) \geq 3$, and deduce a contradiction by finding a homogeneous ideal of height $\operatorname{pr}-\operatorname{dim}(A)-1$ that requires a generating set of cardinality $\operatorname{pr}-\operatorname{dim}(A)$. By Proposition 3.5, we may suppose that $\operatorname{pr}-\operatorname{dim}(A)=3$.

We use without mention the fact that $A$ is a standard graded CohenMacaulay affine domain, together with the well-known properties of such domains, such as their being catenary. Note that these are the only hypotheses (together with the fact that $\operatorname{char}(k)=0$ ) used in the remainder of the argument. That is, we prove the following: 
A standard graded Cohen-Macaulay affine domain over a field of characteristic 0 and of Krull dimension 4 has a relevant homogeneous prime ideal $\mathfrak{p}$ of height 2 with $\mu(\mathfrak{p})=3$.

Step 1. Constructing the prototype of the prime ideal we seek. Since $\mathrm{k}-\operatorname{dim}(A)=4$, using Proposition 3.4 we can choose a regular sequence $x, y, z$ of homogeneous elements of degree 1 in $A$. Set $u=x^{2}-y z, v=y^{2}-x z$, and $w=z^{2}-x y$, and let $I=(u, v, w) A$; note that $u, v, w$ are homogeneous of degree 2. It is easily seen that $u, v, w$ are all nonzero elements; for example, note that $\operatorname{rad}((u, y) A)=\operatorname{rad}((x, y) A)$; hence, $(u, y) A$ has height 2 , so $u \neq 0$.

By way of motivation, we note in passing that $I$ is an almost complete intersection Northcott ideal ([N], [Va]), and it is also a determinantal ideal. (The ideal analyzed in Section 2 also had these properties, and they will prove crucial in the analysis of the ideal $K$ that we will focus on below.) We sketch the details as follows.

Setting

$$
\Phi=\left(\begin{array}{cc}
x & z \\
-z & -y
\end{array}\right)
$$

and letting $\top$ denote matrix transpose, we have that

$$
\Phi \cdot\left(\begin{array}{ll}
x & -y
\end{array}\right)^{\top}=\left(\begin{array}{ll}
u & v
\end{array}\right)^{\top}
$$

and that $w=\operatorname{det}(\Phi)$. Note that $u, v$ is a regular sequence, since $u$ is a nonzero element in the domain $A$ and $v$ cannot lie in any associated prime of $u$ (which is necessarily at height 1 ), because $(u, v, z) A$ has height 3 as a consequence of the equality $\operatorname{rad}((u, v, z) A)=\operatorname{rad}((x, y, z) A)$. It follows from [OP, Section 2], since $(u, v) A$ has grade 2, that $I$ is grade-unmixed of height 2. Note also that

$$
I=I_{2}\left(\begin{array}{lll}
x & y & z \\
y & z & x
\end{array}\right)
$$

so, given the properties of $I$ stated above, the graded version of the HilbertBurch theorem provides us with the resolution

$$
0 \rightarrow A^{2}(-2) \stackrel{\varphi_{2}}{\rightarrow} A^{3}(-1) \stackrel{\varphi_{1}}{\rightarrow} A \rightarrow A / I \rightarrow 0
$$

where

$$
\varphi_{1}=\left(\begin{array}{lll}
w & u & v
\end{array}\right) \quad \text { and } \quad \varphi_{2}^{\top}=\left(\begin{array}{lll}
x & y & z \\
y & z & x
\end{array}\right)
$$


so this resolution is then minimal. Hence, $\mu(I)=3$. We also see, using the Auslander-Buchsbaum formula, that $I_{\mathcal{M}}$ and hence $I$ is a Cohen-Macaulay ideal. (For further properties of such ideals, see [OP, Sections 2 and 5].)

We now use and develop [SUV, Theorem 4.6] along similar lines, in the present setting.

Consider the polynomial extension $D:=A[X, Y]$; note that $D$ is a standard graded Cohen-Macaulay domain, on giving each of $X$ and $Y$ the weighting 1. Set

$$
K=(u+x X, v+x Y) D:_{D} x .
$$

In $(3)$, on premultiplying by $\operatorname{adj}(\Phi)$, one obtains the relationship

$$
x w=-y u-z v .
$$

One easily checks that, as a result, $w-y X-z Y \in K$. We wish to show that $K$ is a prime ideal of $D$, that $K=L:=(u+x X, v+x Y, w-y X-z Y) D$, and that $\mu(K)=3$. (Note that $K$ is then a homogeneous ideal of $D$, being generated by quadrics.) The ideal $K$ is the prototype of the prime ideal in $A$ that we seek so as to establish our contradiction.

We first show that $u+x X$ is a prime element in $D$. Note that, as remarked above, $u$ is a regular element in the domain $A$, since $u \neq 0$. Next, $x$ is regular modulo $u A$; otherwise, $(u, x) A$ would have height 1 , which would contradict the fact that $\operatorname{rad}((u, x) A)(=\operatorname{rad}((x, y) A) \cap \operatorname{rad}((x, z) A))$ has height 2 . That $u+x X$ is a prime element in $A[X]$ follows from [Ka, Exercise 3, p. 102]; hence, $u+x X$ is also a prime element in $D$. Now $v+x Y \notin(u+x X) D$, since $v \notin u A$, and we deduce that $u+x X, v+x Y$ is a regular sequence in $D$. Note also that $K$ is a proper ideal in $D$, since otherwise it would follow that $x \in$ $(u, v) A$, which would contradict the fact that $x$ is actually regular modulo the larger ideal $I \equiv(u, v, w) A$. Hence, $K$ is unmixed of height 2 , by basic properties of the colon operation vis-à-vis finite intersections and primary ideals. Since $w-y X-z Y \in K$, it is easily seen that, up to radical, $K+x D$ contains the elements $x, y, z(z-Y)$. Since each of the ideals $(x, y, z) D$ and $(x, y, z-Y) D$ has height 3 , it follows that $K+x D$ has height 3 and hence that $x$ is regular modulo $K$, since $K$ is unmixed of height 2 . But it is clear that $(D / K)_{x}$ is isomorphic to $A_{x}[(u / x),(v / x)]$, that is, to the domain $A_{x}$. Hence, $D / K$ is a domain, so $K$ is indeed a prime ideal.

Recall the ideal $L:=(u+x X, v+x Y, w-y X-z Y) D$ introduced above. Let

$$
\Psi=\left(\begin{array}{cc}
x+X & -z \\
-z+Y & y
\end{array}\right) .
$$


Note that $\Psi \cdot\left(\begin{array}{ll}x & y\end{array}\right)^{\top}=(u+x X \quad v+x Y)^{\top}$ and that $\operatorname{det}(\Psi)=-(w-y X-$ $z Y)$. We have seen above that $\operatorname{grade}((u+x X, v+x Y) D)=2$ and that $K$ (and so $L$ ) is a proper ideal. Hence, by [N, Theorem 2], the projective dimension of $D / L$ is 2 , and $L$ is grade and hence height unmixed, with all its associated primes having height 2 . The argument above that established that $K+x D$ had height 3 actually showed that $L+x D$ had height 3 . Hence, $x$ is regular modulo $L$.

Now

$$
(u+x X, v+x Y) D \subseteq L \subseteq K \equiv(u+x X, v+x Y) D:_{D} x,
$$

so that, on localizing at each associated prime $\mathfrak{p}$ of $L$, we have

$$
(u+x X, v+x Y) D_{\mathfrak{p}} \subseteq L_{\mathfrak{p}} \subseteq K_{\mathfrak{p}} \equiv(u+x X, v+x Y) D_{\mathfrak{p}}
$$

Hence, $L_{\mathfrak{p}}=K_{\mathfrak{p}}$ for all such $\mathfrak{p}$, so $L=K$. Thus,

$$
K=(u+x X, v+x Y, w-y X-z Y) D .
$$

So

$$
K=I_{2}\left(\begin{array}{ccc}
x & z & -y \\
y & x+X & -z+Y
\end{array}\right),
$$

and again by the Hilbert-Burch theorem, on setting

$$
\begin{aligned}
\psi_{1} & =\left(\begin{array}{ccc}
w-y X-z Y & v+x Y & -(u+x X)
\end{array}\right), \\
\psi_{2}^{\top} & =\left(\begin{array}{ccc}
x & z & -y \\
y & x+X & -z+Y
\end{array}\right),
\end{aligned}
$$

we have the minimal free resolution

$$
0 \rightarrow D^{2}(-2) \stackrel{\psi_{2}}{\rightarrow} D^{3}(-1) \stackrel{\psi_{1}}{\rightarrow} D \rightarrow D / K \rightarrow 0
$$

of $D / K$. In particular, $\mu(K)=3$, and as before, an application of the Auslander-Buchsbaum formula shows that $K$ is a Cohen-Macaulay ideal.

So $K$ has the features that we are seeking - a homogeneous prime ideal of height 2 , minimally generated by three elements - except that the projective variety $\mathbb{V}_{+}(K)$ (consisting set-theoretically of all relevant homogeneous prime ideals containing $K)$ lies not in $\operatorname{Proj}(A)$ but in $\operatorname{Proj}(A[X, Y])$. We now employ a double use of a Bertini theorem to project back into $\operatorname{Proj}(A)$. 
Step 2. Retracting back into A via the double use of a Bertini theorem. For a standard graded Cohen-Macaulay algebra $S$ of positive Krull dimension, note that the scheme $\operatorname{Proj}(S)$ is integral if and only if $S$ is an integral domain. We use this fact below without further comment.

In our setting then, the scheme $\operatorname{Proj}(D / K)$ is integral. Note that pr$\operatorname{dim}(D / K)=3$. Now apply $\left[\mathrm{F},(5.5)\right.$ Satz], with the role of the $f_{i}$ there being played by the images of $x_{1}, \ldots, x_{n}, X, Y$ in $D / K$, each of which has grade 1 . We abuse notation by continuing to write $x_{1}, \ldots, x_{n}, X, Y$ for these respective images. Hence, in applying [F, (5.5) Satz], the scheme $D_{+}\left(f_{0}, \ldots, f_{n}\right)$ featured there in fact equals all of $\operatorname{Proj}(D / K)$. (Recall that $D_{+}\left(f_{0}, \ldots, f_{n}\right)$ consists set-theoretically of all relevant homogeneous prime ideals that do not contain $\left\{f_{0}, \ldots, f_{n}\right\}$.)

We now make the elementary observation that if $R$ is a ring and $r$ is an element of $R$, then, for an indeterminate $Z$ over $R$, we have a natural induced isomorphism $R[Z] /(Z-r) \approx R$ arising from the natural retraction on $R[Z]$ that maps $Z$ to $r$ (and is the identity map on $R$ ). We apply this observation to a generic hyperplane section $H$, say, of $\operatorname{Proj}(D / K)$ and then to a further generic hyperplane section $H^{\prime}$, say, of $\operatorname{Proj}(D / K) \cap H$, resulting finally in an integral subscheme of $\operatorname{Proj}(A)$.

Hence, by $[\mathrm{F},(5.5) \mathrm{Satz}]$, for all $\alpha:=\left(\alpha_{1}, \ldots, \alpha_{n+2}\right)$ in a nonempty Zariskiopen set in $k^{n+2}$, and following the standard notation in [F, Section 5], $\mathbb{V}_{+}\left(f_{\alpha}\right)$ is an integral subscheme of $\operatorname{Proj}(D / K)$, where $f_{\alpha}:=\alpha_{1} x_{1}+\cdots+$ $\alpha_{n} x_{n}+\alpha_{n+1} X+\alpha_{n+2} Y$. Without loss of generality, we may suppose that $\alpha_{n+2} \neq 0$, using the intersection of nonempty Zariski-open sets in $k^{n+2}$. Note that $K$ is generated by quadrics, so that the variety $\mathbb{V}_{+}(K)$ is nondegenerate (i.e., is not contained in a hyperplane). Set

$$
Y^{\prime}=-\left(\alpha_{1} x_{1}+\cdots+\alpha_{n} x_{n}+\alpha_{n+1} X\right) / \alpha_{n+2}, \quad D^{\prime}=A[X]
$$

By our elementary observation, therefore, $K^{\prime}:=\left(u+x X, v+x Y^{\prime}, w-y X-\right.$ $\left.z Y^{\prime}\right) D^{\prime}$ is a homogeneous prime ideal which is generated by quadrics, and $\operatorname{pr}-\operatorname{dim}\left(D^{\prime} / K^{\prime}\right)=2$.

Fix such an $\alpha$ in $k^{n+2}$. Proceeding as before, there exists a nonempty Zariski-open set in $k^{n+1}$, depending on $\alpha$, such that for all $\beta:=\left(\beta_{1}, \ldots, \beta_{n+1}\right)$ in this set, we have $\beta_{n+1} \neq 0$, and on setting

$$
X^{\prime}=-\left(\beta_{1} x_{1}+\cdots+\beta_{n} x_{n}\right) / \beta_{n+1}
$$


and

$$
Y^{\prime \prime}=-\left(\alpha_{1} x_{1}+\cdots+\alpha_{n} x_{n}+\alpha_{n+1} X^{\prime}\right) / \alpha_{n+2},
$$

$K^{\prime \prime}:=\left(u+x X^{\prime}, v+x Y^{\prime \prime}, w-y X^{\prime}-z Y^{\prime \prime}\right) A$ is a prime ideal with $\operatorname{pr}-\operatorname{dim}\left(A / K^{\prime \prime}\right)=1$, so $\operatorname{grade}\left(K^{\prime \prime}\right)=\operatorname{height}\left(K^{\prime \prime}\right)=2$. Now the presentation

$$
K=I_{2}\left(\begin{array}{ccc}
x & z & -y \\
y & x+X & -z+Y
\end{array}\right)
$$

carries over to give the equality

$$
K^{\prime \prime}=I_{2}\left(\begin{array}{ccc}
x & z & -y \\
y & x+X^{\prime} & -z+Y^{\prime \prime}
\end{array}\right),
$$

and hence the resolution (4) carries over to give the minimal presentation

$$
0 \rightarrow A^{2}(-2) \stackrel{\psi_{2}^{\prime}}{\rightarrow} A^{3}(-1) \stackrel{\psi_{1}^{\prime}}{\rightarrow} A \rightarrow A / K^{\prime \prime} \rightarrow 0
$$

where

$$
\begin{aligned}
\psi_{1}^{\prime} & =\left(\begin{array}{ccc}
w-y X^{\prime}-z Y^{\prime \prime} & v+x Y^{\prime \prime} & -\left(u+x X^{\prime}\right)
\end{array}\right), \\
\left(\psi_{2}^{\prime}\right)^{\top} & =\left(\begin{array}{ccc}
x & z & -y \\
y & x+X^{\prime} & -z+Y^{\prime \prime}
\end{array}\right) .
\end{aligned}
$$

In particular, $\mu\left(K^{\prime \prime}\right)=3$, so $K^{\prime \prime}$ is the prime ideal we seek, and this yields the desired contradiction.

Remark 3.7. Note that this result is optimal. Consider the ring $A$ where $A$ is the polynomial ring $k[X, Y, Z]$, with $k$ algebraically closed, under the natural grading. We see from the projective Nullstellensatz and the fact that $A$ is a UFD that $A$ is a gr-Shimoda ring, and $\operatorname{pr}-\operatorname{dim}(A)=2$.

\section{$\S 4$. More technical results in the standard graded case}

We can adapt arguments used in [Mi] to prove the following results. We provide only some additional details, as necessary.

Theorem 4.1 (see [Mi, Corollary 2.7 (and Theorem 2.1)]). Let $S$ be a standard graded algebra over a field $k$ of characteristic 0 , such that $S$ is a domain, $\mathrm{k}-\operatorname{dim}(S) \geq 4$, and $S$ satisfies the Serre conditions $\left(R_{2}\right)$ and $\left(S_{3}\right)$. Suppose further that homogeneous prime ideals of $S$ of height 1 are principal. Then $S$ possesses height 2 homogeneous prime ideals requiring an arbitrarily large number of generators. 
Sketch of Proof. Let $M$ be the graded second syzygy in a minimal graded free resolution of a finitely generated graded $S$-module of finite projective dimension at least 3 , so that $M$ itself is not free. Note that by the EvansGriffith syzygy theorem (see [BH, Corollary 9.5.6 and subsequent remark]), $\operatorname{rank}(M) \geq 2$. Let $d=\operatorname{rank}(M)-1$.

Suppose that we have the graded presentation $S^{c} \stackrel{\alpha}{\rightarrow} S^{b} \rightarrow M \rightarrow 0$ (ignoring twists). Set $A=S\left[Y_{i, j} \mid 1 \leq i \leq b, 1 \leq j \leq d\right]$, giving each indeterminate $Y_{i, j}$ over $S$ weight 1 . Define $\phi: A^{d} \rightarrow A^{b}$ by the matrix $\left(Y_{i, j}\right)$. Let $J=\operatorname{coker}(\alpha \oplus \phi)$. Hence, we have the presentation

$$
A^{c} \oplus A^{d \stackrel{\alpha \oplus \phi}{\rightarrow}} A^{b} \rightarrow J \rightarrow 0 .
$$

Clearly, $\operatorname{rank}(J)=1$. Note that $N:=M \otimes A \simeq A^{b} / \alpha\left(A^{c}\right)$ as graded modules, so there is an exact sequence

$$
0 \rightarrow A^{d} \stackrel{\psi}{\rightarrow} N \rightarrow J \rightarrow 0
$$

with the graded homomorphism $\psi$ induced by $\phi$.

A straightforward adaptation of Miller's argument [Mi, p. 31] then shows that the graded module $J$ is a torsion-free $A$-module and so, as is easily seen, it is a homogeneous ideal (up to graded isomorphism).

Next we show that we may suppose that the homogeneous ideal $J$ has height 2. Suppose that $J$ has height 1 . By an obvious "homogeneous" analogue of the proof of [Ka, Theorem $5(\mathrm{~b})]$, given our hypotheses, a nonzero homogeneous element of $A$ that is not a unit is a unique product of homogeneous elements each of which generates a homogeneous prime ideal. (We note that, since $A$ is a domain, a product of nonzero elements of $A$ is homogeneous if and only if each element is homogeneous.) Hence, taking a finite set of homogeneous generators of $J$, we can find a smallest homogeneous principal ideal $a A$, with $a$ a homogeneous element, that contains $J$. Clearly, $a^{-1} J$ is a homogeneous ideal. We claim that $a^{-1} J$ is of height at least 2 . Otherwise, $a^{-1} J$ is contained in a homogeneous prime $P$ of height 1 , and by hypothesis, $P=b A$ for some homogeneous element $b \in A$; it would then follow that $J \subseteq a b A \subseteq a A$, so $a b A=a A$, that is, $b A=A$, a contradiction. Hence, we may suppose that $J$ has height at least 2 . Another straightforward adaptation of Miller's argument [Mi, pp. 31-32] shows that $J$ has precisely height 2 and that $J$ is in fact prime.

Finally, on replacing $A$ by its localization at its irrelevant maximal ideal $\mathcal{M}$, we can directly apply the argument in [Mi] to prove his Corollary 2.7 
with reference to the ideal $\bar{J}:=J_{\mathcal{M}}+\left(Z_{1}, \ldots, Z_{b d}\right) A_{\mathcal{M}}$ considered in $S_{\mathcal{N}}$, where $\mathcal{N}$ denotes the irrelevant maximal ideal in $S$ (see [Mi, pp. 33-34]); note that if $x_{1}, \ldots, x_{n}$ are the degree 1 generators of $\mathcal{N}$, then the $Z_{l}$ are general $k$-linear combinations of $x_{1}, \ldots, x_{n}$ and the $Y_{i, j}$, and so are homogeneous forms of degree 1 . We next observe that a standard graded algebra $D$ with irrelevant maximal ideal $\mathcal{P}$ is a domain if $D_{\mathcal{P}}$ is a domain. It follows that the homogeneous ideal $\widetilde{J}:=J+\left(Z_{1}, \ldots, Z_{b d}\right) A$, considered in $S$, is a prime ideal. Since $\widetilde{J}$ requires at least as many generators as $\bar{J}$, the result follows.

The arguments in the proof of Step 1 of the proof of Theorem 3.6 can easily be adapted for use in the following more general situation. We adopt the notation of Section 3 .

THEOREM 4.2. Let $A$ be a standard graded algebra over a field of characteristic 0, with $A$ a domain. Suppose that k-dim $(A) \geq 4$ and that $A$ satisfies the Serre condition $\left(S_{3}\right)$. Then $A$ possesses a homogeneous prime ideal $P$ of height 2 with $\mu(P)=3$.

Sketch of Proof. In the light of [BH, Propositions 1.5.15(e), 1.5.11, and 1.5.12], a straightforward adaptation of the original argument means that we need only comment on how to adapt the details of Step 2, namely, the application of the double use of a Bertini theorem, in order to finish the proof.

Replace $D$ by its localization at its irrelevant ideal, say $\mathcal{M}^{\prime}$, and $A$ by its localization at its irrelevant ideal $\mathcal{M}$.

As before, $D / K$ has projective dimension 2. Since $A$ satisfies the condition $\left(S_{3}\right)$ and $X, Y$ are indeterminates over $A$, $\operatorname{depth}(D) \geq 5$. By the Auslander-Buchsbaum formula, therefore, $\operatorname{depth}(D / K) \geq 3$. We can now mimic the argument in the second part of the proof of [Mi, Theorem 2.1, p. 33], here cutting with two generic hyperplanes $Z_{1}$ and $Z_{2}$, in the notation at [Mi, p. 33].

By Flenner's Bertini theorem (see [Mi, p. 32]), $D /\left(K+Z_{1} D\right)$ is analytically irreducible. Note that $\operatorname{depth}\left(D /\left(K+Z_{1} D\right)\right) \geq 2$. Hence, another application of Bertini's theorem is allowed, and we have that $D /\left(K+\left(Z_{1}, Z_{2}\right) D\right)$ is again analytically irreducible.

From the short exact sequence $0 \rightarrow D \stackrel{Z_{1}}{\rightarrow} D \rightarrow D / Z_{1} D \rightarrow 0$, we quickly establish that $\operatorname{Tor}_{i}^{D}\left(D / K, D / Z_{1} D\right)=0$ for $i \geq 1$. Hence, the minimal free resolution of $D / K$ over $D$ afforded by the Hilbert-Burch theorem descends via tensoring with $D / Z_{1} D$ to give the minimal free resolution over $D / Z_{1} D$ 
of $D /\left(K+Z_{1} D\right)$, this being in Hilbert-Burch form. Repeating the argument on further factoring out $Z_{2} \cdot D / Z_{1} D$ shows that

$$
\mu_{D /\left(Z_{1}, Z_{2}\right) D}\left(\left(K+\left(Z_{1}, Z_{2}\right) D\right) /\left(Z_{1}, Z_{2}\right) D\right)=3
$$

and the full result now follows.

REMARK 4.3. Suppose that we have a standard graded domain $S$ over a field $k$ such that every homogeneous prime ideal of height 1 (resp., 2) is generated by 1 (resp., 2) elements. It easily follows from the first part of the proof of [BH, Theorem 1.5.9] that $S$ satisfies the condition $\left(R_{2}\right)$. Hence, if we further suppose that $\operatorname{pr}-\operatorname{dim}(S) \geq 3$ and that $k$ has characteristic 0 , then $S$ satisfies the hypotheses of the Theorem 4.1. It follows that Theorem 3.6 is a consequence of Theorem 4.1 .

On the other hand, it is clear that Theorem 3.6 is also a consequence of Theorem 4.2 .

Acknowledgments. We would like to thank J.-M. Giral for drawing the paper [Mi] to our attention and Abdó Roig for useful discussions.

\section{REFERENCES}

[BH] W. Bruns and J. Herzog, Cohen-Macaulay Rings, Cambridge Stud. Adv. Math. 39, Cambridge University Press, Cambridge, 1993. MR 1251956.

[D] E. D. Davis, Ideals of the principal class, R-sequences and a certain monoidal transformation, Pacific J. Math. 20 (1967), 197-205. MR 0206035.

[Di] J. Dieudonné, Topics in Local Algebra, Notre Dame Math. Lect. 10, University of Notre Dame Press, Notre Dame, 1967. MR 0241408.

[E] D. Eisenbud, Commutative Algebra, with a View toward Algebraic Geometry, Grad. Texts in Math. 150, Springer, New York, 1995. MR 1322960. DOI 10. 1007/978-1-4612-5350-1.

[F] H. Flenner, Die Sätze von Bertini für lokale Ringe, Math. Ann. 229 (1977), 97-111. MR 0460317.

[HIO] M. Herrmann, S. Ikeda, and U. Orbanz, Equimultiplicity and Blowing Up: An Algebraic Study, with an appendix by B. Moonen, Springer, Berlin, 1988. MR 0954831. DOI 10.1007/978-3-642-61349-4.

[HS] C. Huneke and I. Swanson, Integral Closure of Ideals, Rings, and Modules, London Math. Soc. Lecture Note Ser. 336, Cambridge University Press, Cambridge, 2006. MR 2266432.

[Ka] I. Kaplansky, Commutative Rings, rev. ed., University of Chicago Press, Chicago, 1974. MR 0345945.

[KS] K. Kiyek and J. Stückrad, Integral closure of monomial ideals on regular sequences, Rev. Mat. Iberoamericana 19 (2003), 483-508. MR 2023197. DOI 10. $4171 / \mathrm{RMI} / 359$. 
[Ma1] H. Matsumura, Commutative Algebra, 2nd ed., Math. Lect. Note Ser. 56, Benjamin/Cummings, Reading, Mass., 1980. MR 0575344.

[Ma2] , Commutative Ring Theory, translated from the Japanese by M. Reid, Cambridge Stud. Adv. Math. 8, Cambridge University Press, Cambridge, 1986. MR 0879273.

[Mi] M. Miller, Bourbaki's theorem and prime ideals, J. Algebra 64 (1980), 29-36. MR 0575778. DOI 10.1016/0021-8693(80)90129-5.

[N] D. G. Northcott, A homological investigation of a certain residual ideal, Math. Ann. 150 (1963), 99-110. MR 0146206.

[OP] L. O'Carroll and F. Planas-Vilanova, Ideals of Herzog-Northcott type, Proc. Edinb. Math. Soc. (2) 54 (2011), 161-186. MR 2764414. DOI 10.1017/ S0013091509001321.

[Sa] J. D. Sally, Numbers of Generators of Ideals in Local Rings, Dekker, New York, 1978. MR 0485852.

[Sh] Y. Shimoda, "On power stable ideals and symbolic power ideals" in Proceedings of the Third Japan-Vietnam Joint Seminar on Commutative Algebra, Hanoi, 2007, 90-95.

[SUV] A. Simis, B. Ulrich, and W. V. Vasconcelos, Jacobian dual fibrations, Amer. J. Math. 115 (1993), 47-75. MR 1209234. DOI 10.2307/2374722.

[St] R. P. Stanley, Hilbert functions of graded algebras, Adv. Math. 28 (1978), 57-83. MR 0485835.

[VV] P. Valabrega and G. Valla, Form rings and regular sequences, Nagoya Math. J. 72 (1978), 93-101. MR 0514892.

[Va] W. V. Vasconcelos, Computational Methods in Commutative Algebra and Algebraic Geometry, Algorithms Comput. Math. 2, Springer, Berlin, 1998. MR 1484973. DOI 10.1007/978-3-642-58951-5.

Shiro Goto

Department of Mathematics

School of Science and Technology

Meiji University

Tama-Ku, Kawasaki-shi

214-8571 Japan

goto@math.meiji.ac.jp

Liam O'Carroll

Maxwell Institute for Mathematical Sciences

School of Mathematics

University of Edinburgh

EH9 3JZ, Edinburgh

Scotland

L. O'Carroll@ed.ac.uk 
Francesc Planas-Vilanova

Departament de Matemàtica Aplicada 1

Universitat Politècnica de Catalunya

08028 Barcelona

Catalunya

francesc.planas@upc.edu 